\title{
Identification and validation of PSAT1 as a potential prognostic factor for predicting clinical outcomes in patients with colorectal carcinoma
}

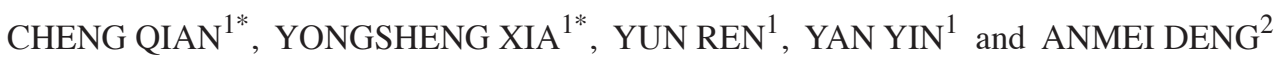 \\ ${ }^{1}$ Department of General Surgery, Huzhou Maternity and Child Care Hospital, Huzhou, Zhejiang 313000; \\ ${ }^{2}$ Department of Laboratory Diagnosis, Changhai Hospital, Second Military Medical University, Shanghai 200433, P.R. China
}

Received December 18, 2015; Accepted August 23, 2017

DOI: $10.3892 / \mathrm{ol} .2017 .7211$

\begin{abstract}
The aim of the present study was to explore the existence of known or candidate drug-target genes that are upregulated in colorectal cancer (CRC) and may serve as novel prognostic factors or therapeutic targets for this type of malignancy. An in silico analysis was conducted using the Oncomine tool to compare the expression levels of a list of drug-target genes between cancerous and normal tissues in 6 independent CRC cohorts retrieved from the Oncomine database. Phosphoserine aminotransferase 1 (PSAT1) was identified as the top-ranked upregulated gene in CRC tumors, and was highly expressed in patients with chemoresistant disease. Subsequently, the expression of PSAT1 was further experimentally validated using immunohistochemistry in an independent cohort of CRC specimens. The immunohistochemistry results demonstrated that PSAT1 was overexpressed in the CRC tissues compared with the normal colorectal tissues, which was consistent with the previous in silico analysis. Furthermore, PSAT1 overexpression was associated with response to irinotecan, 5-fluorouracil and leucovorin chemotherapy, and with shorter survival time, and retained significance as an independent prognostic factor for CRC when subjected to the multivariate analysis with a Cox's proportional hazards model. Therefore, the present results implicate PSAT1 as a potential prognostic biomarker and a
\end{abstract}

Correspondence to: Dr Cheng Qian, Department of General Surgery, Huzhou Maternity and Child Care Hospital, Huzhou, Zhejiang 313000, P.R. China

E-mail: hzqiancheng@sina.com

Dr Anmei Deng, Department of Laboratory Diagnosis, Changhai Hospital, Second Military Medical University, Shanghai 200433, P.R. China

E-mail: anmeideng@aliyun.com

*Contributed equally

Key words: phosphoserine aminotransferase 1, colorectal cancer, chemotherapy resistance, drug target, microarray, prognosis promising therapeutic target for CRC. Targeted PSAT1 inhibition in the treatment of CRC warrants further investigation.

\section{Introduction}

Colorectal cancer (CRC) is the third most frequently occurring cancer, and remains the second most common cause of cancer-associated mortality worldwide $(1,2)$. Even with the use of standard or enhanced treatments, a relatively high proportion of patients develop drug resistance, consequently suffering recurrence and relapse (3). In recent years, several potential therapeutic targets have been used in clinical practice; however, only a small subset of patients with CRC are able to benefit from undergoing treatment with drugs against such targets (4). This situation highlights the importance of identifying novel therapeutic targets that may be exploited for CRC treatment.

Although the identification of novel targets is attractive for cancer treatment, the processes by which this identification is achieved are often tedious and have a high failure rate. Recently, a 'repurposing' strategy has been proposed, in which established non-cancer drug targets are evaluated for anticancer activity (5). This strategy provides an opportunity to rapidly develop and clinically trial novel therapeutic strategies against cancer. For example, using the gene expression data from the Oncomine database, Rhodes et al (6) identified that an anti-hypertensive drug target, angiotensin II receptor type I (AGTR1), was overexpressed in a subset of breast cancer tissues, which demonstrated sensitivity to the AGTR1 antagonist losartan.

In the present study, using a similar strategy, the different expression profiles of approved or candidate drug-target genes between CRC and normal colorectal tissues were evaluated. Phosphoserine aminotransferase 1 (PSAT1), a serine biosynthesis-related target gene, was identified to be specifically and reproducibly overexpressed in CRC tumors, and its expression was associated with resistance to irinotecan, 5-fluorouracil and leucovorin (FOLFIRI) chemotherapy. Subsequently, it was experimentally validated that PSAT1 is significantly associated with a lack of response to chemotherapy and poor clinical outcomes in an independent cohort of CRC samples. 


\section{Materials and methods}

In silico comparison of mRNA expression profiles of food and drug administration (FDA)-approved and literature-defined drug-target genes. Initially, mining of published microarray studies on CRC mRNA expression was performed using the Oncomine tool (www.oncomine.org) as described previously (7). A total of $6 \mathrm{CRC}$ mRNA expression datasets deposited in the Oncomine database were included in the systematic comparative analysis, according to the following filtering criteria available within the database: i) CRC pathological types; ii) clinical specimens; and iii) primary $\mathrm{CRC}$ and normal colorectal samples included. The 6 datasets that met these criteria were analyzed further. Information on these 6 datasets is summarized in Table I (8-13). In addition to the common clinical and pathological characteristics (including tumor size, lymph node metastasis and distant metastasis) included in each dataset, chemotherapy response information was available in the Graudens colon dataset, and survival data were available in The Cancer Genome Atlas (TCGA) CRC dataset.

Subsequently, an in silico analysis was performed to compare the differentially expressed drug-target genes (2-fold variation; $\mathrm{P}<0.0001)$ between $\mathrm{CRC}$ and normal colorectal tissues across the 6 independent CRC datasets, implementing the 'Concepts: Drugbank Targets-FDA approved-Literature-defined' and 'Analysis Type: Cancer vs. Normal Analysis' functions available in the Oncomine database (7). Rank is used to compare the over- or underexpressed genes using the Oncomine tools as described previously (7). To determine the expression pattern of PSAT1, CRC datasets were further filtered by 'Gene: PSAT1'. The expression of PSAT1 in each histological type was also examined, and the associations between PSAT1 expression levels and the clinical and pathological characteristics of the patients in each dataset were analyzed.

CRC specimens for immunohistochemistry (IHC). Formalin-fixed and paraffin-embedded specimens from 88 patients with CRC who underwent surgical resection at Huzhou Maternity and Child Care Hospital (Huzhou, China) between January 2007 and October 2013 were included in the present study, following the acquisition of informed consent. No patient received neo-adjuvant chemotherapy or radiotherapy. In 30 cases, chemotherapy with the FOLFIRI regimen was administered due to the occurrence of metastasis. Drug response was determined according to criteria described previously (14). Briefly, metastatic lesions were evaluated using computed tomography scanning, and cases with a $\geq 50 \%$ decrease in lesion size were classified as responders, whereas patients with a decrease of $<50 \%$ or with an increase in lesion size were classified as non-responders. In all cases, complete data regarding clinicopathological characteristics and follow-up were available, as well as an adequate volume of tumor specimen for IHC. The disease-specific survival time was defined as the interval between surgical resection and mortality due to cancer. All procedures used in the present study were approved by the research ethics committee of Huzhou Maternity \& Child Care Hospital.
$I H C$. Tissue sections (4- $\mu \mathrm{m}$ thick) were subjected to immunohistochemical staining with an EnVision avidin-biotin-peroxidase complex kit (Dako; Agilent Technologies, Inc., Santa Clara, CA, USA). Briefly, following deparaffinization and rehydration, $10 \mathrm{mM}$ citrate buffer at pH 6.0 was used for antigen retrieval at $100^{\circ} \mathrm{C}$ for $10 \mathrm{~min}$. Hydrogen peroxide (3\%) was used to block the activity of endogenous peroxidases. The slides were then stained with primary antibody (rabbit anti-PSAT1 antibody; cat. no. sc-133929; Santa Cruz Biotechnology, Inc., Santa Cruz, CA, USA; dilution, 1:100) at $4^{\circ} \mathrm{C}$ overnight, followed by secondary staining by the chromogenic substrate 3,3'-diaminobenzidine, followed by counterstaining with hematoxylin using the EnVision kit according to the manufacturer's protocol. For the negative control, anti-PSAT1 antibody was replaced by non-specific rabbit IgG (at 1:100 dilution; cat. no. SC2027; Santa Cruz Biotechnology, Inc.). The staining was evaluated by two independent pathologists under a light microscope (magnification, $\mathrm{x} 40$ ) by calculating H-scores, obtained by multiplying the percentage and staining intensity scores in $>10$ fields, as described previously (15). Cases with a score of more or less than the median value were defined as having high or low expression, respectively.

Statistical analysis. The associations between PSAT1 expression levels and clinicopathological features were evaluated using a $\chi^{2}$ test. Kaplan-Meier estimator curves and log-rank test were used for the survival analysis. Multivariate survival analyses were performed using the Cox's proportional hazards model. $\mathrm{P}<0.05$ was considered to indicate a statistically significant difference. All statistical analyses were performed using MedCalc software (version 15.22; MedCalc Software bvba, Ostend, Belgium).

\section{Results}

PSAT1 is identified as the most upregulated drug target gene using in silico analysis. An in silico analysis of the 6 published microarray datasets was performed to determine which drug-target genes were reproducibly upregulated in CRC tumors vs. normal colorectal tissues. Using mRNA gene expression datasets from the Oncomine database, a panel of drug targets that were significantly overexpressed in CRC tumors compared with normal colorectal tissues was identified (Fig. 1). Among this target list, several genes have previously been validated as CRC therapeutic targets, such as vascular endothelial growth factor A (16). Notably, PSAT1, a key serine biosynthesis enzyme, ranked top of the upregulated targets (median rank, 430.5; $\mathrm{P}=6.24 \times 10^{-7}$ ) across all $\mathrm{CRC}$ cohorts (Fig. 2A-F). Furthermore, PSAT1 was not only overexpressed in the whole set of CRC tissues, but also significantly upregulated in each histological subtype in the Kaiser colon and TCGA CRC datasets compared with the normal colorectal tissues (Fig. 2E and F).

Clinical and prognostic value of PSAT1 across CRC datasets. The clinical and prognostic significance of PSAT1 expression in the 6 Oncomine CRC datasets was evaluated. No association between PSAT1 and clinical traits was observed, except that patients with CRC who did not exhibit 
Table I. Colorectal cancer mRNA expression datasets used in the present study.

\begin{tabular}{lllrr}
\hline Oncomine name & GEO ID & \multicolumn{1}{c}{ Platform } & Cases, n & (Refs.) \\
\hline Gaedcke colorectal & GSE20842 & Agilent Human Genome 44K & 130 & $(8)$ \\
Graudens colon & GSE3964 & 11K_VJF-ARRAY & 60 & $(9)$ \\
Hong colorectal & GSE9348 & Human Genome U133 Plus 2.0 Array & 82 & $(10)$ \\
Kaiser colon & GSE5206 & Human Genome U133 Plus 2.0 Array & 105 & $(11)$ \\
Ki colon & GSE6988 & Human 17K cDNA-GeneTrack & 123 & $(12)$ \\
TCGA colorectal & - & Agilent 244K Custom Gene Array Illumina RNA-Seq & 237 & $(13)$ \\
\hline
\end{tabular}

TCGA, The Cancer Genome Atlas; GEO, Gene Expression Omnibus; ID, identifier.

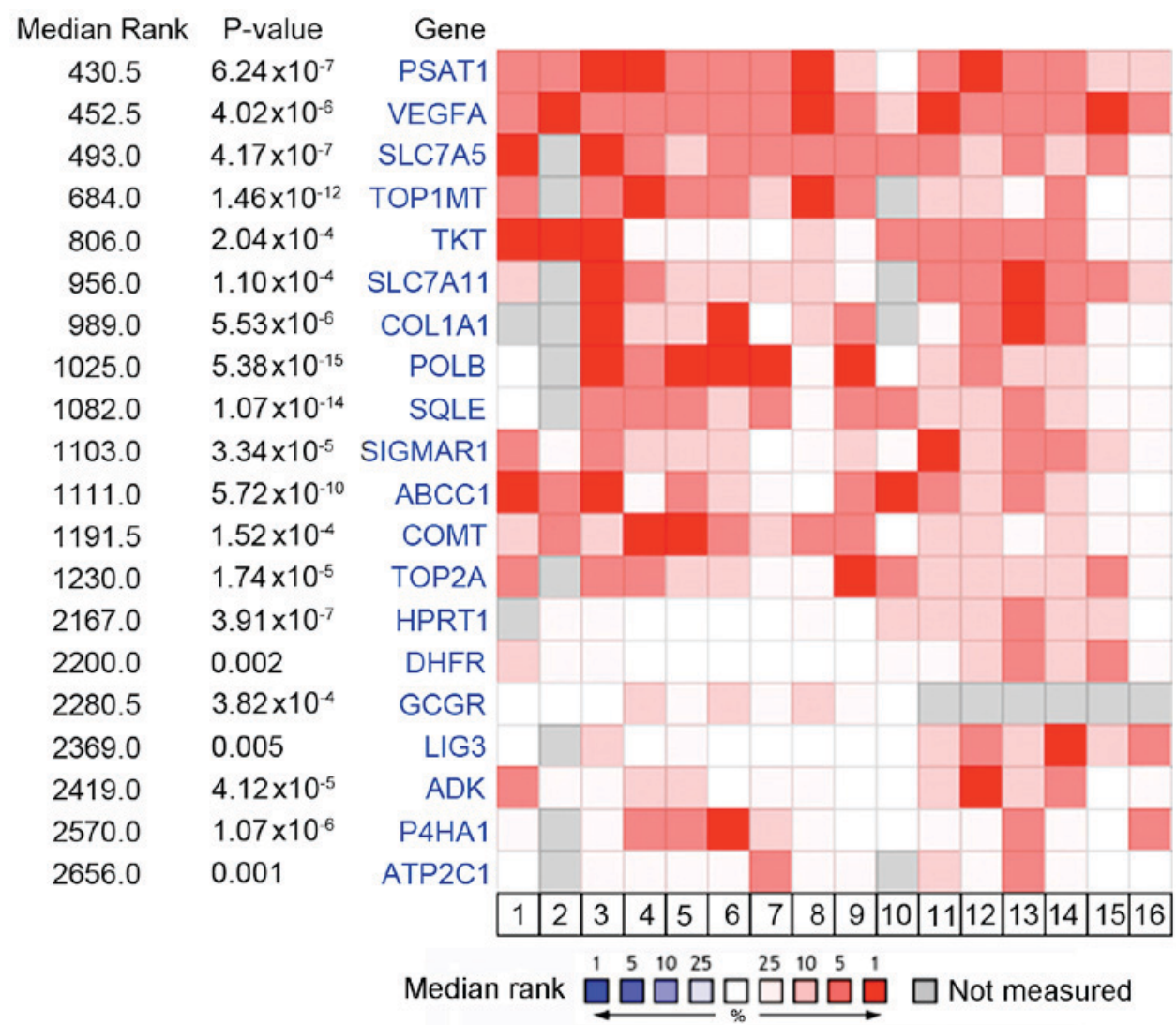

Figure 1. Comparison of gene expression of approved or candidate drug targets between CRC and normal colorectal tissues across mRNA gene expression databases from the Oncomine database. PSAT1 ranked top of the upregulated targets in CRC tissues across different CRC datasets and histology subtypes. CRC, colorectal cancer; PSAT1, phosphoserine aminotransferase 1.

a response to FOLFIRI chemotherapy had an increased PSAT1 expression level compared with those who did exhibit a response (Graudens colon dataset); and patients who were alive without tumor at 3 years exhibited relatively decreased PSAT1 expression compared with those who were alive with tumor and deceased at 3 years (TCGA CRC dataset) (Fig. 2G and H).

PSAT1 is a biomarker and prognostic factor for CRC. Immunohistochemical validation of the expression pattern and clinical significance of PSAT1 was performed in an independent CRC cohort. The IHC results identified that PSAT1 staining was positive in the cytoplasm and membrane of cancer cells (Fig. 3A). As expected, PSAT1 staining was observed in CRC tissues with various degrees of intensity and density, whereas no staining was detected in tumor-adjacent normal colorectal tissues, which is consistent with the results of the in silico analysis. Heterogeneous PSAT1 expression among CRC tumors was observed. On the basis of the median H-score, the 88 cases were divided into high- and low-expression subgroups. PSAT1 expression was identified to be associated with FOLFIRI treatment efficacy; as presented in Table II, PSAT1 overexpression was more frequently observed in FOLFIRI non-responders $(84.2 \%)$ than in FOLFIRI responders $(36.4 \%)(\mathrm{P}=0.0228)$. No significant association with other clinical characteristics was identified.

Furthermore, survival data analysis revealed that a high expression level of PSAT1 was associated with poor 


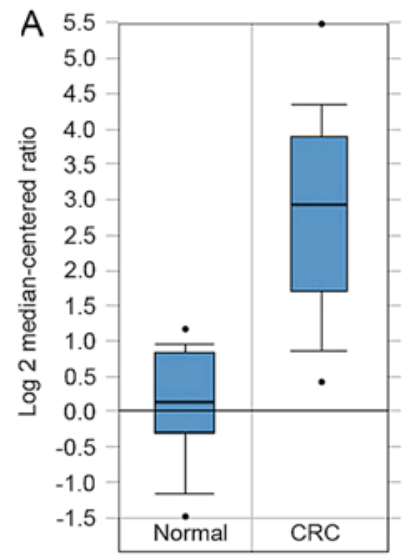

Hong colorectal

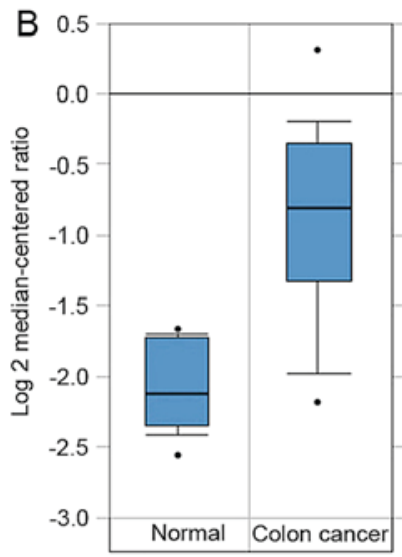

Graudens colon

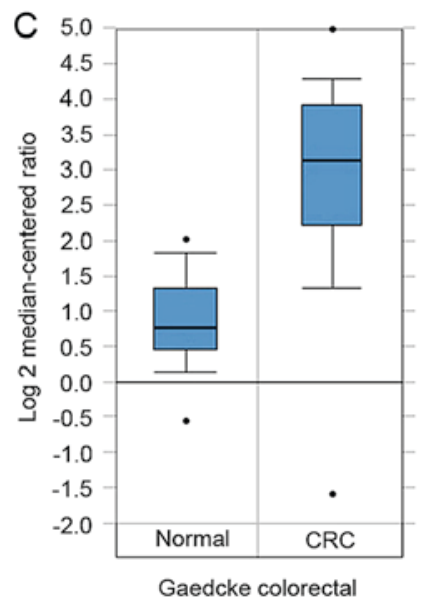

Gaedcke colorectal

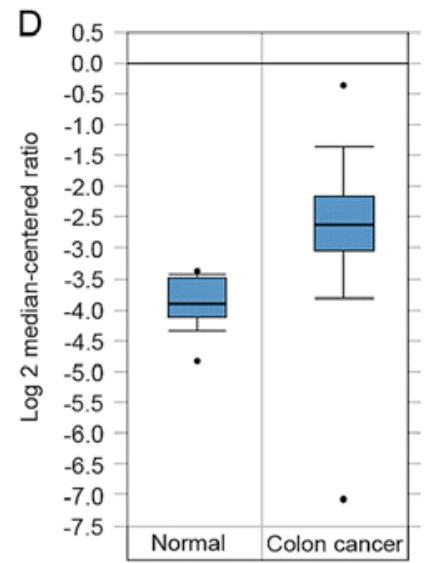

Ki colon

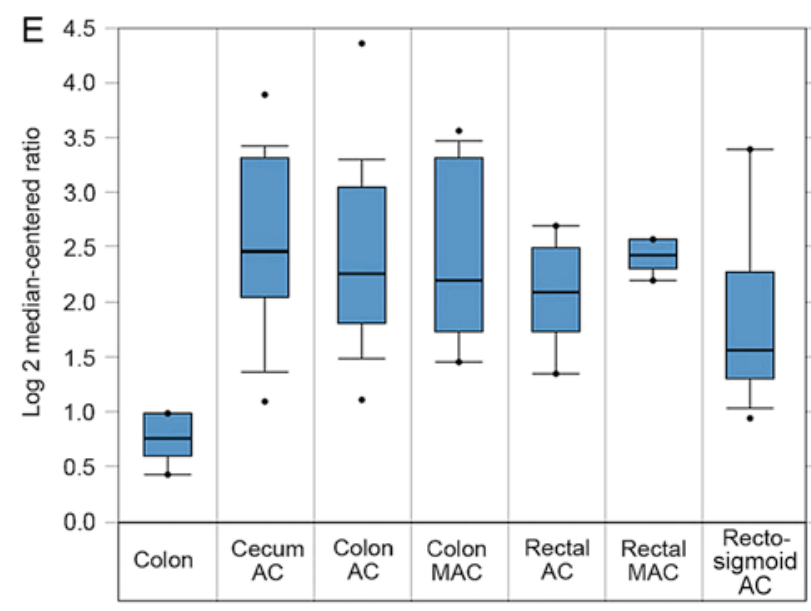

Kaiser colon
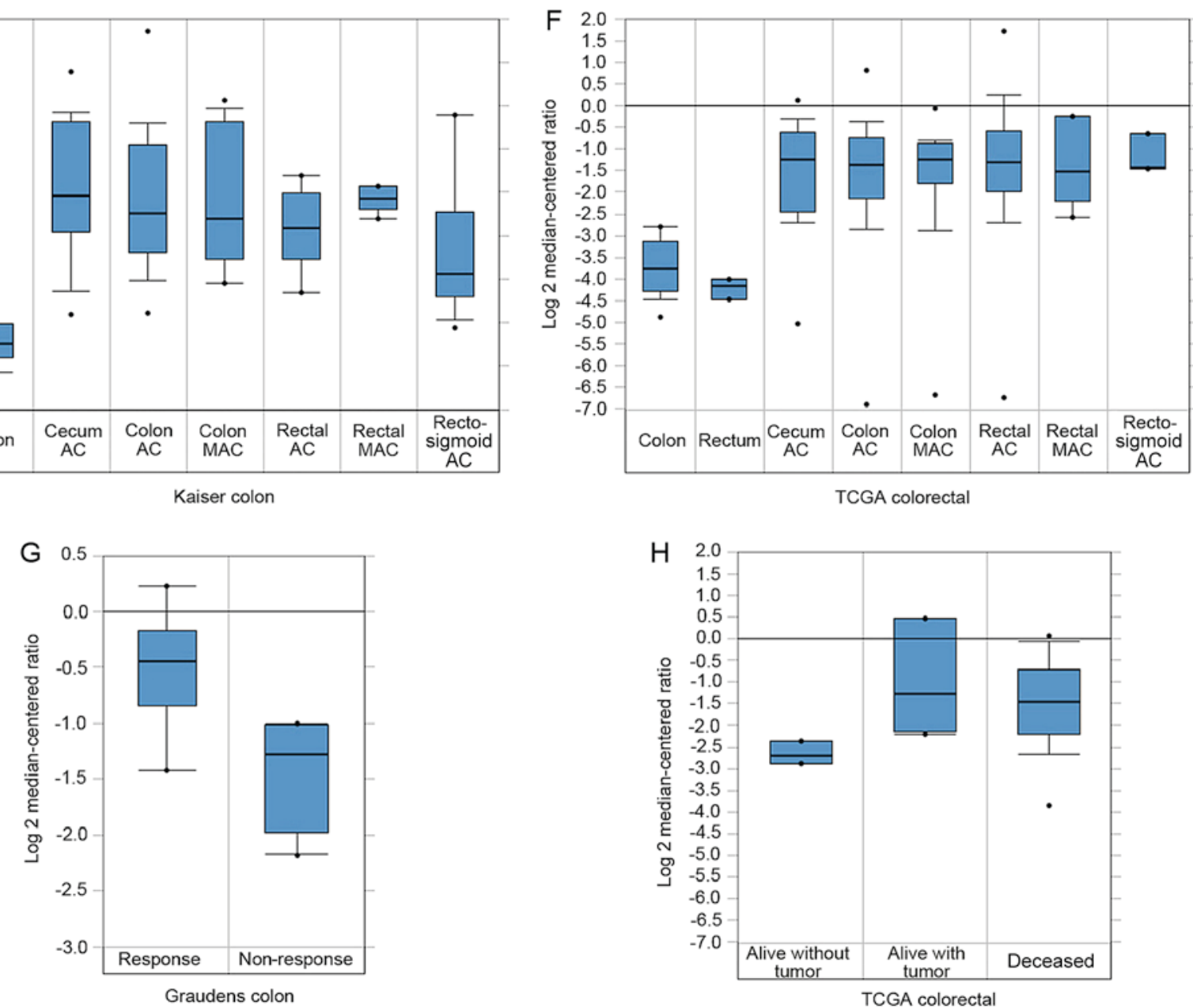

Figure 2. Expression of PSAT1 in 6 colorectal datasets from the Oncomine database. PSAT1 was upregulated in the whole set of CRC tissues compared with normal controls in 4 databases: (A) Hong colorectal; (B) Graudens colon; (C) Gaedcke colorectal; and (D) Ki colon. It was also overexpressed in different histological subtypes in (E) the Kaiser colon dataset and (F) TCGA colorectal dataset. (G) In the Graudens colon dataset, cases without FOLFIRI response exhibited increased PSAT1 expression level compared with those with FOLFIRI response. (H) Poor prognosis subgroups (deceased or alive with tumor after 3 years of follow-up) exhibited an increased expression level of PSAT1 compared with the favorable prognosis subgroup (alive without tumor). CRC, colorectal cancer; PSAT1, phosphoserine aminotransferase 1; TCGA, The Cancer Genome Atlas; FOLFIRI, irinotecan, 5-fluorouracil and leucovorin; AC, adenocarcinoma; MAC, mucinous adenocarcinoma.

disease-specific survival [hazard ratio (HR), 2.1802; 95\% confidence interval (CI), 1.2289-3.8680; $\mathrm{P}=0.008$, log-rank test; Fig. 3B; Table III], and was identified as an independent prognostic factor on multivariate analysis (HR, 2.5950; 95\% CI, 1.4317-4.7035; P=0.0018; Table III).

\section{Discussion}

In the present study, the expression profiles of a list of approved or candidate drug-target genes were compared in silico between cancerous and normal tissues across independent 

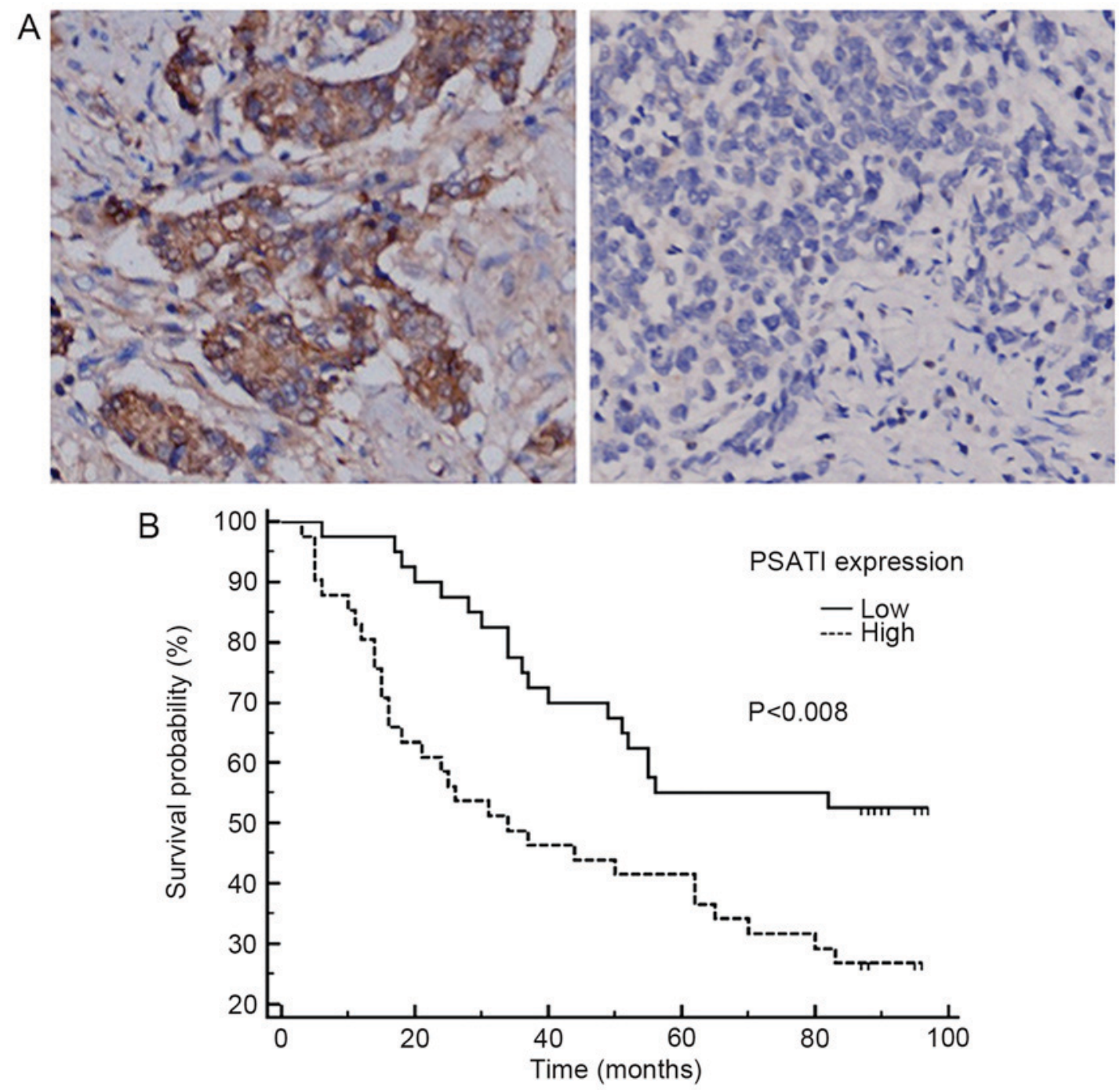

Figure 3. Representative images of PSAT1 immunostaining in colorectal cancer tissues, and Kaplan-Meier estimator curves based on its expression. (A) Strong PSAT1 staining was observed in the cytoplasm and membrane of cancer cells (left), and negative staining was observed in colorectal cancer tissues (right). (B) The high PSAT1 expression subgroup, defined by the median H-score threshold value, demonstrated a shorter overall survival time than that of the low expression subgroup (median, 19.0 vs. 30.0 months; $\mathrm{P}<0.008$, log-rank test). PSAT1, phosphoserine aminotransferase 1.

CRC cohorts. The PSAT1 gene and protein were identified and validated to be reproducibly overexpressed in CRC tumors, and PSAT1 expression levels were identified to be associated with response to FOLFIRI treatment. The analysis also suggested that PAST1 may serve as a potential independent prognostic factor for CRC. To the best of our knowledge, the present study is the first to report the clinicopathological and prognostic significance of PSAT1 in CRC.

PSAT1 encodes a key enzyme involved in serine synthesis, a process that serves a pivotal role in proliferating cancer cells and is associated with clinical aggressiveness (17). Mutation or decreased levels of PSAT1 may be associated with schizophrenia or phosphoserine aminotransferase deficiency. Marked PSAT1 expression has been identified in various cancer types, including breast cancer, and non-small cell lung cancer $(18,19)$. Martens et al $(20)$ reported that DNA methylation of PSAT1 was inversely associated with its mRNA expression and with poor clinical outcome. Furthermore, PSAT1 may be a predictor of tamoxifen therapy response in breast cancer (21). In a previous study of a small population of patients with CRC, using microarray and semi-quantitative PCR methods, Vié et al (22) observed that PSAT1 mRNA was increased in colon tumors compared with normal colorectal tissues. The present study verified and extended these earlier results in clinical setting and a larger cohort of patients, and further confirmed that PSAT1-positive CRC cases may represent a subgroup of more aggressive disease, with resistance to the currently used regimens and with poorer clinical outcomes.

Experimental studies have indicated that inhibiting PSAT1 could aid in the treatment of cancer $(16,22)$. Vié et al (22) also suggested that PSAT1 may be implicated in cancer progression and chemoresistance in human colon cells, reporting that a PSAT1-positive cell line was more resistant to oxaliplatin treatment compared with a non-transfected cell line lacking PSAT1 expression. Additionally, Yang et al (18) found that PSAT1 could sustain the proliferation of non-small cell lung cancer cells by inhibiting cyclin D1 degradation and subsequently activating the retinoblastoma-E2F transcription 
Table II. Comparison of clinicopathological characteristics between high and low PSAT1 expression subgroups evaluated by immunohistochemistry.

\begin{tabular}{|c|c|c|c|c|}
\hline \multirow[b]{2}{*}{ Characteristics } & \multirow[b]{2}{*}{ Total patients, $\mathrm{n}$} & \multicolumn{2}{|c|}{ PSAT1 expression } & \multirow[b]{2}{*}{ P-value } \\
\hline & & Low, $\mathrm{n}$ & High, $n$ & \\
\hline Age, years & & & & 0.6043 \\
\hline$<60$ & 19 & 11 & 8 & \\
\hline$\geq 60$ & 69 & 33 & 36 & \\
\hline Sex & & & & 0.5220 \\
\hline Male & 46 & 21 & 25 & \\
\hline Female & 42 & 23 & 19 & \\
\hline Tumor grade & & & & 0.1016 \\
\hline 1 & 10 & 4 & 6 & \\
\hline 2 & 46 & 28 & 18 & \\
\hline 3 & 32 & 12 & 20 & \\
\hline Tumor size & & & & 0.4817 \\
\hline $\mathrm{T} 1-\mathrm{T} 2$ & 9 & 3 & 6 & \\
\hline T3-T4 & 79 & 41 & 38 & \\
\hline Lymph node metastasis & & & & 0.8267 \\
\hline Negative & 54 & 27 & 27 & \\
\hline Positive & 34 & 17 & 17 & \\
\hline Distant metastasis & & & & 0.4744 \\
\hline Negative & 86 & 43 & 43 & \\
\hline Positive & 2 & 1 & 1 & \\
\hline Stage & & & & 0.8267 \\
\hline $1-2$ & 54 & 27 & 27 & \\
\hline $2-4$ & 34 & 17 & 17 & \\
\hline FOLFIRI treatment & & & & 0.0228 \\
\hline Response & 11 & 7 & 4 & \\
\hline No response & 19 & 3 & 16 & \\
\hline
\end{tabular}

PSAT1, phosphoserine aminotransferase 1; FOLFIRI, irinotecan, 5-fluorouracil and leucovorin.

Table III. Univariate and multivariate analysis for overall survival according to PSAT1 expression evaluated immunohistochemistry.

\begin{tabular}{|c|c|c|c|c|}
\hline \multirow[b]{2}{*}{ Prognostic factor } & \multicolumn{2}{|c|}{ Univariate analysis } & \multicolumn{2}{|c|}{ Multivariate analysis } \\
\hline & $\operatorname{HR}(95 \% \mathrm{CI})$ & $\mathrm{P}$-value & $\mathrm{HR}(95 \% \mathrm{CI})$ & P-value \\
\hline Tumor grade & $1.4765(0.9477-2.3003)$ & 0.0865 & $1.3006(0.8007-2.1126)$ & 0.2907 \\
\hline Tumor size & $1.2658(0.5037-3.1807)$ & 0.6179 & $1.6396(0.5110-5.2613)$ & 0.4083 \\
\hline Lymph node metastasis & $2.3543(1.3400-4.1364)$ & 0.0030 & $1.9354(1.0478-3.5751)$ & 0.0359 \\
\hline Distant metastasis & $9.8512(2.2493-43.1446)$ & 0.0025 & $22.8051(3.4643-150.1243)$ & 0.0012 \\
\hline PSAT1 expression & $2.1802(1.2289-3.8680)$ & 0.0080 & $2.5950(1.4317-4.7035)$ & 0.0018 \\
\hline
\end{tabular}

PSAT1, phosphoserine aminotransferase 1; HR, hazard ratio; CI, confidence interval.

factor signaling pathway. On the basis of the present and previous results, we hypothesize that PSAT1 may be a prognostic biomarker as well as a promising therapeutic target, and that a PSAT1-positive CRC subgroup may benefit from therapies directed against this target. Investigation of PSAT1 inhibition in animal models and development of small-molecule inhibitors of PSAT1 merit attention in future research.

In summary, the present study identified and validated the overexpression of PSAT1 in CRC tumors compared with normal colorectal tissues, and demonstrated that its 
overexpression is associated with response to chemotherapy and poor clinical outcome. Therefore, it is possible that PSAT1 may be used as a prognostic marker and a potential therapeutic target for patients with CRC.

\section{Acknowledgements}

The present study was supported by the National Science Foundation of China (grant no. 81471605), a Shanghai Shenkang Grant (grant no. CHDC22014014) and Changhai Hospital (grant no. CH125530300).

\section{References}

1. Siegel RL, Miller KD and Jemal A: Cancer statistics, 2015. CA Cancer J Clin 65: 5-29, 2015.

2. Zheng R, Zeng H, Zhang S, Chen $\mathrm{T}$ and Chen W: National estimates of cancer prevalence in China, 2011. Cancer Lett 370 : 33-38, 2016.

3. Kanwar SS, Poolla A and Majumdar AP: Regulation of colon cancer recurrence and development of therapeutic strategies. World J Gastrointest Pathophysiol 15: 1-9, 2012.

4. Meyerhardt JA and Mayer RJ: Systemic therapy for colorectal cancer. N Engl J Med 352: 476-487, 2005.

5. Gupta SC, Sung B, Prasad S, Webb LJ and Aggarwal BB: Cancer drug discovery by repurposing: Teaching new tricks to old dogs. Trends Pharmacol Sci 34: 508-517, 2013.

6. Rhodes DR, Ateeq B, Cao Q, Tomlins SA, Mehra R, Laxman B, Kalyana-Sundaram S, Lonigro RJ, Helgeson BE, Bhojani MS, et al: AGTR1 overexpression defines a subset of breast cancer and confers sensitivity to losartan, an AGTR1 antagonist. Proc Natl Acad Sci USA 106: 10284-10289, 2009.

7. Rhodes DR, Kalyana-Sundaram S, Mahavisno V, Varambally R, Yu J, Briggs BB, Barrette TR, Anstet MJ, Kincead-Beal C, Kulkarni P, et al: Oncomine 3.0: Genes, pathways, and networks in a collection of 18,000 cancer gene expression profiles. Neoplasia 9: 166-180, 2007.

8. Gaedcke J, Grade M, Jung K, Camps J, Jo P, Emons G, Gehoff A, Sax U, Schirmer M, Becker H, et al: Mutated KRAS results in overexpression of DUSP4, a MAP-kinase phosphatase, and SMYD3, a histone methyltransferase, in rectal carcinomas. Genes Chromosomes Cancer 49: 1024-1034, 2010.

9. Graudens E, Boulanger V, Mollard C, Mariage-Samson R, Barlet X, Grémy G, Couillault C, Lajémi M, Piatier-Tonneau D, Zaborski $\mathrm{P}$, et al: Deciphering cellular states of innate tumor drug responses. Genome Bio 7: R19, 2006.

10. Hong Y, Downey T, Eu KW, Koh PK and Cheah PY: A 'metastasis-prone' signature for early-stage mismatch-repair proficient sporadic colorectal cancer patients and its implications for possible therapeutics. Clin Exp Metastasis 27: 83-90, 2010.
11. Kaiser S, Park YK, Franklin JL, Halberg RB, Yu M, Jessen WJ, Freudenberg J, Chen X, Haigis K, Jegga AG, et al: Transcriptional recapitulation and subversion of embryonic colon development by mouse colon tumor models and human colon cancer. Genome Bio 8: R131, 2007.

12. Ki DH, Jeung HC, Park CH, Kang SH, Lee GY, Lee WS, Kim NK, Chung HC and Rha SY: Whole genome analysis for liver metastasis gene signatures in colorectal cancer. Int J cancer 121: 2005-2012, 2007.

13. Cancer Genome Atlas Network: Comprehensive molecular characterization of human colon and rectal cancer. Nature 487: 330-337, 2012.

14. Therasse P, Arbuck SG, Eisenhauer EA, Wanders J, Kaplan RS, Rubinstein L, Verweij J, Van Glabbeke M, van Oosterom AT, Christian MC and Gwyther SG: New guidelines to evaluate the response to treatment in solid tumors. J Natl Cancer Inst 92: 205-216, 2000.

15. Huang HJ, Neven P, Drijkoningen M, Paridaens R, Wildiers H, Van Limbergen E, Berteloot P, Amant F, Vergote I and Christiaens MR: Association between tumour characteristics and HER-2/neu by immunohistochemistry in 1362 women with primary operable breast cancer. J Clin Path 58: 611-616, 2005.

16. Khan K, Cunningham D and Chau I: Targeting angiogenic pathways in colorectal cancer: Complexities, challenges and future directions. Curr Drug Targets 18: 56-71, 2017.

17. Antonov A, Agostini M, Morello M, Minieri M, Melino G and Amelio I: Bioinformatics analysis of the serine and glycine pathway in cancer cells. Oncotarget 5: 11004-11013, 2014.

18. Yang Y, Wu J, Cai J, He Z, Yuan J, Zhu X, Li Y,Li M and Guan H: PSAT1 regulates cyclin D1 degradation and sustains proliferation of non-small cell lung cancer cells. Int J Cancer 136: E39-E50, 2015.

19. Kim YH, Jung WH and Koo JS: Expression of metabolism-related proteins in invasive lobular carcinoma: Comparison to invasive ductal carcinoma. Tumor Bio 35: 10381-10393, 2014.

20. Martens JW, Nimmrich I, Koenig T, Look MP, Harbeck N, Model F, Kluth A, Bolt-de Vries J, Sieuwerts AM, Portengen $\mathrm{H}$, et al: Association of DNA methylation of phosphoserine aminotransferase with response to endocrine therapy in patients with recurrent breast cancer. Cancer Res 65: 4101-4117, 2005.

21. De Marchi T, Timmermans MA, Sieuwerts AM, Smid M, Look MP, Grebenchtchikov N, Sweep FCGJ, Smits JG, Magdolen V, van Deurzen CHM, et al: Phosphoserine aminotransferase 1 is associated to poor outcome on tamoxifen therapy in recurrent breast cancer. Sci Rep 7: 2099, 2017.

22. Vié N, Copois V, Bascoul-Mollevi C, Denis V, Bec N, Robert B, Fraslon C, Conseiller E, Molina F, Larroque C, et al: Overexpression of phosphoserine aminotransferase PSAT1 stimulates cell growth and increases chemoresistance of colon cancer cells. Mol Cancer 7: 14, 2008. 